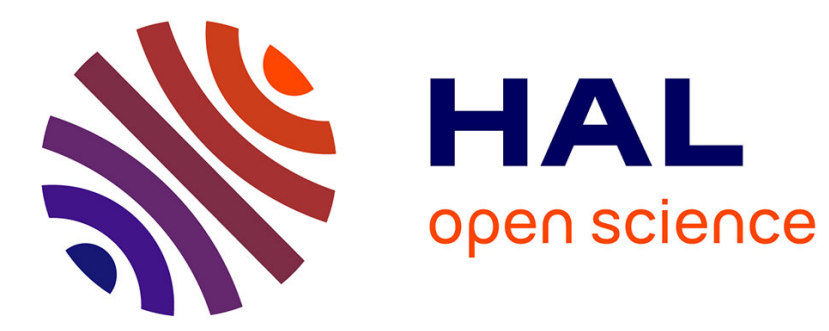

\title{
On the robustness of phase-locking in Kerr optical frequency combs
}

\author{
Aurélien Coillet, Kouomou Yanne Chembo
}

\section{To cite this version:}

Aurélien Coillet, Kouomou Yanne Chembo. On the robustness of phase-locking in Kerr optical frequency combs. Optics Letters, 2014, 39 (6), pp.1529-1532. 10.1364/OL.39.001529 . hal-03222044

\section{HAL Id: hal-03222044 \\ https://hal.science/hal-03222044}

Submitted on 20 May 2021

HAL is a multi-disciplinary open access archive for the deposit and dissemination of scientific research documents, whether they are published or not. The documents may come from teaching and research institutions in France or abroad, or from public or private research centers.
L'archive ouverte pluridisciplinaire HAL, est destinée au dépôt et à la diffusion de documents scientifiques de niveau recherche, publiés ou non, émanant des établissements d'enseignement et de recherche français ou étrangers, des laboratoires publics ou privés. 


\title{
On the robustness of phase locking in Kerr optical frequency combs
}

\author{
Aurélien Coillet* and Yanne Chembo \\ FEMTO-ST Institute [CNRS UMR6174], Optics Department, 16 Route de Gray, 25030 Besançon Cedex, France \\ *Corresponding author: aurelien.coillet@femto-st.fr
}

\begin{abstract}
We theoretically investigate the phase locking phenomena between the spectral components of Kerr optical frequency combs in the dynamical regime of Turing patterns. We show that these Turing patterns display a particularly strong and robust phase locking, originating from a cascade of phase locked triplets which asymptotically lead to a global phase locking between the modes. The local and global phase locking relationships defining the shape of the comb are analytically determined. Our analysis also shows that solitons display a much weaker phase locking that can be destroyed more easily than in the Turing pattern regime. Our results indicate that Turing patterns are generally the most suitable for applications requiring the highest stability. Experimental generation of such combs is also discussed in detail, and is in excellent agreement with the numerical simulations.
\end{abstract}

Kerr optical frequency combs have been the focus of a strong interest from the scientific community since their first generation a few years ago [1]. These combs result from a four-wave mixing process where new frequencies are generated through the nonlinear interaction between the light and the bulk material of a monolithic whispering gallery mode (WGM) resonator. Depending on the frequency and power of the pump laser, this phenomenology leads to the excitation of equally spaced spectral lines, thereby forming the so-called Kerr comb. This multiwavelength optical source is very promising for various applications such as high resolution spectroscopy and ultrastable microwave generation. In the latter case, the generated microwave corresponds to the beating between the different spectral modes, and its phase noise is directly related to the phase relationship between the comb's lines [2-4]. The understanding of the phase locking process and its robustness with regard to external perturbations is therefore of the highest importance for ultrastable microwave generation, and the objective of this Letter is to unveil the mechanisms ensuring a stable and robust phase locking between the modes.

The experimental setup used for Kerr comb generation is shown on Fig. 1. The output of a continuous-wave $1550 \mathrm{~nm}$ laser is amplified and coupled to an ultrahigh $Q$ WGM resonator using a tapered fiber. Above a given pump power threshold, a Kerr comb is generated and can be monitored using an optical spectrum analyzer.

A spatiotemporal formalism based on the LugiatoLefever equation (LLE) has recently been proposed to describe Kerr comb generation in WGM resonators [믁. In our case, the LLE can be written as [ㅁ]

$$
\frac{\partial \psi}{\partial \tau}=-(1+i \alpha) \psi+i|\psi|^{2} \psi-i \frac{\beta}{2} \frac{\partial^{2} \psi}{\partial \theta^{2}}+F
$$

where $\psi(\theta, \tau)$ is the complex amplitude of the overall field in the rotating frame of reference of the cavity, $\theta \in[-\pi, \pi]$ is the azimuthal angle along the cavity circumference, $\tau=t / 2 \tau_{\mathrm{ph}}$ is the normalized time, with $\tau_{\mathrm{ph}}=1 / \Delta \omega_{\mathrm{tot}}$ being the photon lifetime, $\Delta \omega_{\text {tot }}=\omega_{0} / Q_{\text {tot }}$ the loaded linewidth, and $Q_{\text {tot }}$ the loaded quality factor. The parameter $\alpha=-2\left(\Omega-\omega_{0}\right) / \Delta \omega$ is the detuning between the pump laser and the cold resonance frequencies $\Omega$ and $\omega_{0}$, respectively. The parameter $\beta$ represents the second-order dispersion of the resonator, while $F$ stands for the amplitude of the pump laser field.

Various nontrivial solutions can emerge from the LLE, depending on the parameters $\alpha, \beta$ and $F[8,9]$. In the regime of anomalous dispersion, the time-independent stationary solutions are either Turing patterns or bright solitons. Turing patterns correspond to an integer number of intensity extrema along the azimuthal direction of the cavity. In the Fourier domain, they correspond to the well-known Kerr combs with multiple free spectral range (FSR) spacing [10-14].

A few examples of such combs are presented in Fig. 2. They have been generated in a magnesium fluoride $\left(\mathrm{MgF}_{2}\right)$ resonator with intrinsic $Q$-factor equal to $1.5 \times 10^{9}$, and a FSR equal to $\Omega_{\mathrm{FSR}} / 2 \pi=5.86 \mathrm{GHz}$. By choosing different resonances, pump powers, and frequencies we were able to obtain different mode spacings, each corresponding to a different integer number of FSR [11]. For each one of these spectra, numerical simulations were performed using the split-step Fourier method on the LLE, and a good agreement with the experiments was found, hence validating the theoretical description. In the case of Fig. 2(b), the parameters used were $\beta=-0.03, \alpha=1.27$, and $\overline{F^{2}}=1.075$. For Fig. $2(\mathrm{~d})$, $\beta$ is equal to $-0.003, \alpha=-1.3$ and $F^{2}=6.4$, while in Fig. 2(f), $\beta=-0.001, \alpha=-1.87$, and $F^{2}=14$.

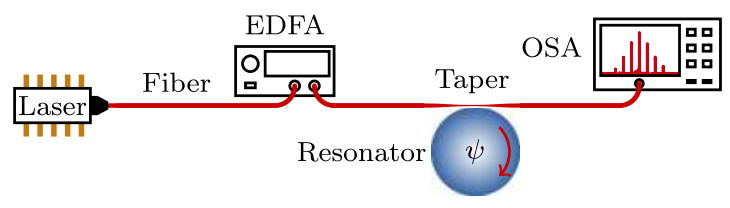

Fig. 1. Experimental setup. 

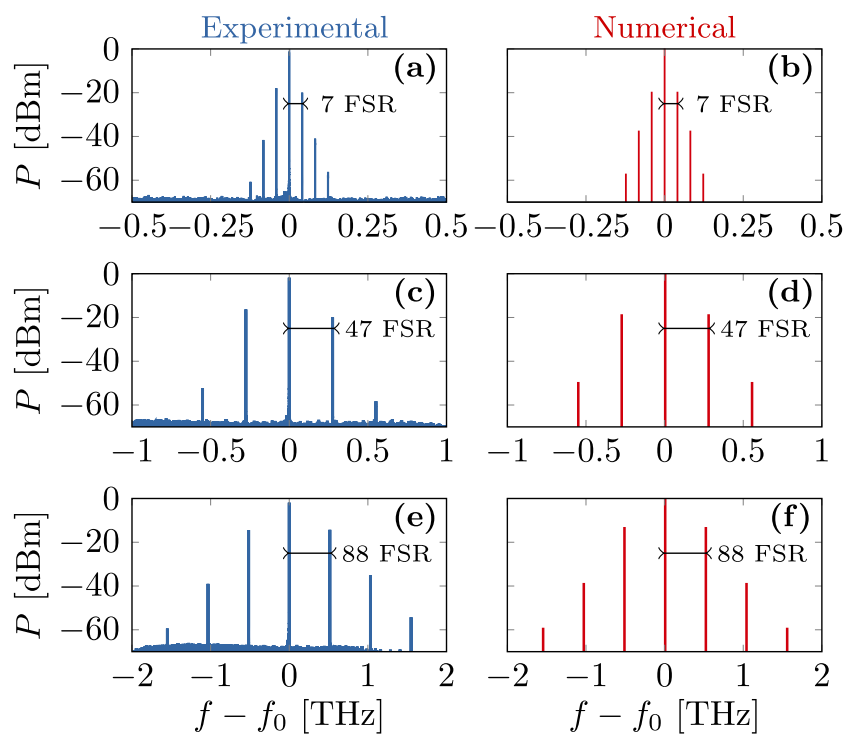

Fig. 2. Comparison between experimental [(a), (c), and (e)] and theoretical [(b), (d), and (f)] multiple-FSR spectra of Kerr combs corresponding to Turing patterns. Various line spacings are observed ranging from 7 to 88 FSR $(41-516 \mathrm{GHz})$.

To investigate theoretically the phase locking of Turing patterns and their robustness relatively to solitons, an intermediate set point was chosen, with $\beta=-0.03, \alpha=0$, $F^{2}=2.5$, and noisy initial conditions, leading to the generation of the 12th order Turing pattern shown in Fig. 3(a). Contrary to Turing patterns that entirely fill the cavity, the bright cavity soliton is a unique structure spinning in the cavity [14]. The spectrum of the soliton is therefore composed of spectral lines separated by only one FSR, as shown on Fig. $3(\mathrm{~b})[\alpha=3, \beta=-0.03$, $F^{2}=3$; noisy Gaussian pulse initial condition]. Cavity solitons are known to be subcritical dissipative structures and, in the LLE, they appear for values of $\alpha$ greater than $41 / 30$.

Turing patterns and cavity solitons are the two most promising Kerr comb regimes for ultrapure microwave generation. They are, indeed, structurally stable solutions of the LLE and can be obtained with parameter values far from chaotic regimes. In this application, the microwave signal corresponds to the beating between the different modes of the comb detected by a fast photodiode. To evaluate the phase properties of this beat note one needs to investigate the relative phases of the different modal components of the comb. From an analytical point of view this task can hardly be performed using the LLE. Instead, the following modal decomposition,

$$
\psi(\theta, \tau)=\sum_{l} \Psi_{l}(\tau) e^{i l \theta}
$$

allows direct access to the phase, $\varphi(\tau)$, of the slowly varying modal amplitudes, $\Psi_{l}=\left|\Psi_{l}\right| e^{i \varphi}$, with $l=\ell-\ell_{0}$ being the azimuthal eigennumber of the photons with respect to the pumped mode (the pumped mode is therefore $l=0$, while the sidemodes correspond to $l= \pm 1, \pm 2, \ldots$ ). By plugging the expansion of Eq. (2) inside the LLE, the following ordinary differential equations ruling the dynamics of each mode are obtained:

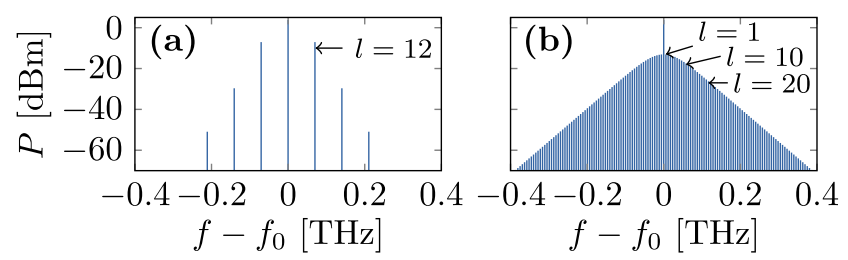

Fig. 3. Simulated spectra of (a) a Turing pattern with $L=12$ and (b) a cavity soliton.

$$
\begin{aligned}
\frac{d \Psi_{l}}{d \tau}= & {\left[-(1+i \alpha)+i \frac{\beta}{2} l^{2}\right] \Psi_{l}+\delta(l) F } \\
& +i \sum_{m, n, p} \delta(m-n+p-l) \Psi_{m} \Psi_{n}^{*} \Psi_{p}
\end{aligned}
$$

where $\delta(x)$ is the usual Kronecker function, while $m, n, p$, and $l$ are eigennumbers labeling the interacting modes following the interaction $\hbar \omega_{m}+\hbar \omega_{p} \leftrightarrow$ $\hbar \omega_{n}+\hbar \omega_{l}$. Note that strict equivalence between the modal and spatiotemporal models had been demonstrated [ [6], so that the theoretical analysis can be performed with either formalism depending on their specific advantages.

The regime of the Turing pattern is essentially originating from modulational instability, which was analyzed in detail in Refs. [12-17]. Turing patterns actually arise from the following cascade of interactions. When the pump is set above the threshold $F_{\text {th }}^{2}=1+(1-\alpha)^{2}$, the modes around $\pm l_{\mathrm{th}}$, with $l_{\mathrm{th}}=[(2(\alpha-2) / \beta)]^{1 / 2}$, experience positive gain and are thereby excited. After transient dynamics characterized by modal competition, two symmetric sidemodes, $\pm L \simeq \pm l_{\text {th }}$, are stabilized around the pump and the net flux of photons follows the degenerate interaction $2 \hbar \omega_{0} \rightarrow \hbar \omega_{L}+\hbar \omega_{-L}$. From then, the sidemodes $\pm L$ provide, in their turn, a net flux of photons to the sidemodes $\pm 2 L$ using interactions $\hbar \omega_{0}+\hbar \omega_{ \pm L} \rightarrow \hbar \omega_{\mp L}$ $+\hbar \omega_{ \pm 2 L}$. This cascaded process is further generalized later through the interactions $\hbar \omega_{0}+\hbar \omega_{ \pm L} \rightarrow \hbar \omega_{\mp k L}+$ $\hbar \omega_{ \pm(k+1) L}$ where the sidemodes $\pm k L$ provide a net flux of photons to the sidemodes $\pm(k+1) L$. This cascaded fountain effect asymptotically yields a Kerr comb characterized by a multiple-FSR spacing of multiplicity $L$. The effect of this cascading mechanism can be observed in Fig. 4, displaying the phase dynamics of the 12th order Turing patterns of Fig. 3(a). It can be clearly seen that,

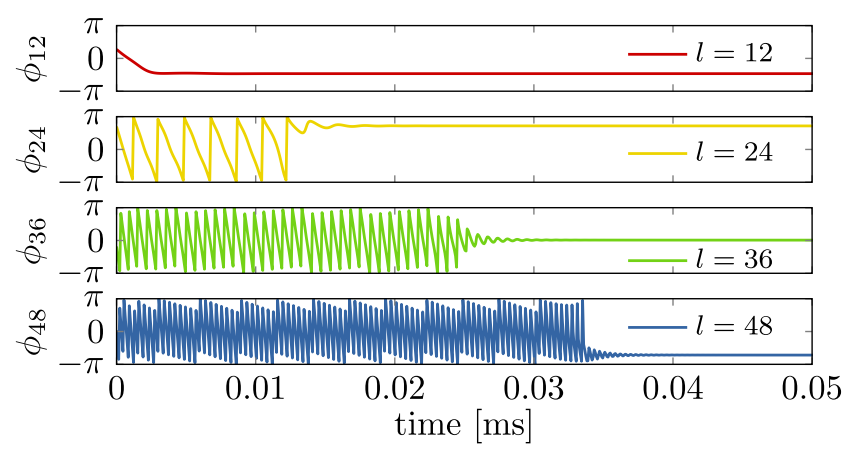

Fig. 4. Time evolution of the relative phases of the 4 first modes of the Turing pattern of Fig. 3(a). After a delay increasing with the mode number, the relative phases reach a constant value, the Kerr comb becoming phase locked. 


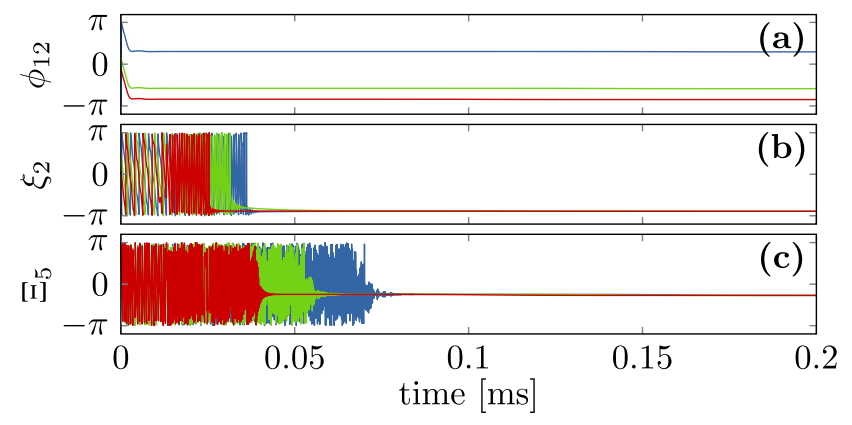

Fig. 5. Phase locking behavior for different noisy initial conditions (represented by different colors). (a) Phase difference, $\phi_{12}=\varphi_{12}-\varphi_{0}$. Evidence of triplet phase locking [(b), $\xi_{2}$ is constant], and global phase locking [(c), $\Xi_{5}$ is constant].

after transient dynamics, the various modal phases, $\phi_{l}=\varphi_{l}-\varphi_{0}$, with respect to the pump are reaching a time-independent value. The phase stabilization occurs first for the mode $l=12$, and then to $l=24, l=36$, and so on.

It is important to note that the phase locking value depends on the initial condition, as illustrated on Fig. 5(a), where the phase difference, $\phi_{l}=\varphi_{l}-\varphi_{0}$ for $l=12$, has been plotted for different initial conditions. This phenomenon is true for all of the excited modes of the Turing pattern. However, it can be shown that an intrinsic phase locking relationship takes place between the modes of the comb. According to the scenario described earlier, a given mode, $k L$, mainly interacts with its two neighboring modes, $(k \pm 1) L$. In the steady state $\left(d \Psi_{k L} / d \tau \equiv 0\right)$, this three-mode interaction can be rewritten as [15]

$$
\begin{aligned}
\kappa_{k L} \Psi_{k L}= & \delta(k) F+\left|\Psi_{k L}\right|^{2} \Psi_{k L} \\
& +2\left\{\left|\Psi_{(k-1) L}\right|^{2} \Psi_{k L}+\left|\Psi_{(k+1) L}\right|^{2} \Psi_{k L}\right. \\
& \left.+\Psi_{(k-1) L} \Psi_{k L}^{*} \Psi_{(k+1) L}\right\},
\end{aligned}
$$

with $\kappa_{l}=-(1+i \alpha)+i \beta l^{2} / 2$. This approximate relationship, obtained from a photon flux balance standpoint, can be used to show that consecutive triplets of modes are necessarily phase locked according to

$$
\xi_{k}=\varphi_{(k-1) L}-2 \varphi_{k L}+\varphi_{(k+1) L}=\text { constant. }
$$

Hence, the phases, $\varphi_{k L}$, might individually have an asymptotic value that depends on the initial conditions but, for the same Turing pattern, the combination $\varphi_{(k-1) L}-2 \varphi_{k L}+\varphi_{(k+1) L}$ will be independent of initial conditions and will converge to the same value, $\xi_{k}$, as can be seen in Fig. $5(\mathrm{~b})$. The value of $\xi_{k}$ depends only on the parameters $\alpha, \beta, F$, and $k$. The sequence is, therefore, the following. Initially, the triplet $\{-L, 0, L\}$ is the first to phase lock. Then, the two triplets $\{0, \pm L, \pm 2 L\}$ phase lock and, later on, all the cascade of triplets $\{ \pm(k-1) L, \pm k L, \pm(k+1) L\}$ as $k \rightarrow+\infty$ successively phase lock as well.

This sequence of triplet phase locking ultimately leads to a global phase coupling through a cascaded process, and the global phase locking relationship can be obtained by considering that since any $\xi_{k}$ is constant so is any linear combination of them. If we consider $2 N+1$ oscillating modes in a Turing pattern comb (the pump and $N$ excited modes on each side), we can show that

$$
\begin{aligned}
\Xi_{N} & =\frac{1}{2} \sum_{k=-(N-1)}^{k=N-1}\left[1-(-1)^{N+k}\right] \xi_{k} \\
& =\varphi_{-N L}+2 \sum_{k=-(N-1)}^{k=N-1}(-1)^{N+k} \varphi_{k L}+\varphi_{N L} \\
& =\text { Constant. }
\end{aligned}
$$

If we consider, for example, the 7 oscillating modes that are visible in Fig. 3(a), this global phase locking relationship simply reads $\Xi_{3}=\varphi_{-36}-2 \varphi_{-24}+2 \varphi_{-12}-2 \varphi_{0}+$ $2 \varphi_{12}-2 \varphi_{24}+\varphi_{36}=$ Constant. This relationship can include more modes, if needed [for example the modes $l= \pm 48$, which are indeed oscillating as it can be seen in Fig. 4(d)]. Hence, when the permanent state is reached, the linear combination, $\Xi_{N}$, which involves all the modal phases is a constant that is independent of the initial conditions, as evidenced by Fig. 5(c). These intrinsic phase relations, $\xi_{k}$ and $\Xi_{N}$, are, therefore, responsible for the shape of the Kerr comb in the Turing pattern regime.

In order to test the robustness of the phase locking in both the Turing pattern and the soliton regimes, we introduce a perturbation after the stationary state is reached. The phase of the pump signal is abruptly shifted by constant offset, and we monitor the difference, $\Delta \phi_{l}(t)=\phi_{l}(t)-\phi_{l}^{\text {st }}$, with $\phi_{l}=\varphi_{l}-\varphi_{0}$. When the offset in the pump phase is set to $\pi / 5$, the relative phases of the different modes oscillate in response to this perturbation, but the phase locking is preserved. In the case of the Turing patterns, the phase oscillations are of small amplitude with respect to the perturbation and decrease when the mode number increases, as if the perturbation was attenuated during its propagation to higher order modes. In contrast, the amplitudes of the phase oscillations of the soliton are larger than the initial stimulus and do not substantially decrease for higher order modes. This result indicates that Turing patterns are more robust to external phase perturbations and could lead to better phase noise performances. To strengthen this claim, the phase shift of the pump signal was increased to $\pi / 2$ and the resulting phase evolution is plotted in Figs. 6(c) and $\underline{6(d)}$. The Turing pattern behavior remains very stable and identically phase locked, while the soliton is destroyed (as well as the phase locking).

In this work, we have given an insight into the dynamics of phase locking in a Kerr frequency comb. The fact that the photonic interactions are strongly dominated by the exchange of microwave photons of energy $L \times \hbar \Omega_{\mathrm{FSR}}$ is a direct consequence of the modulational instability, which generates the highest gain for photons whose offset frequency relative to the pump is precisely $L \times \Omega_{\mathrm{FSR}}$. We have analytically demonstrated that phase locking in Turing patterns is achieved through a cascaded phase locking of adjacent triplets, which ultimately leads to a global phase locking relationship that we have determined. We have also shown that Turing patterns are more robust than solitons with regard to external perturbations, thereby demonstrating their stronger potential 

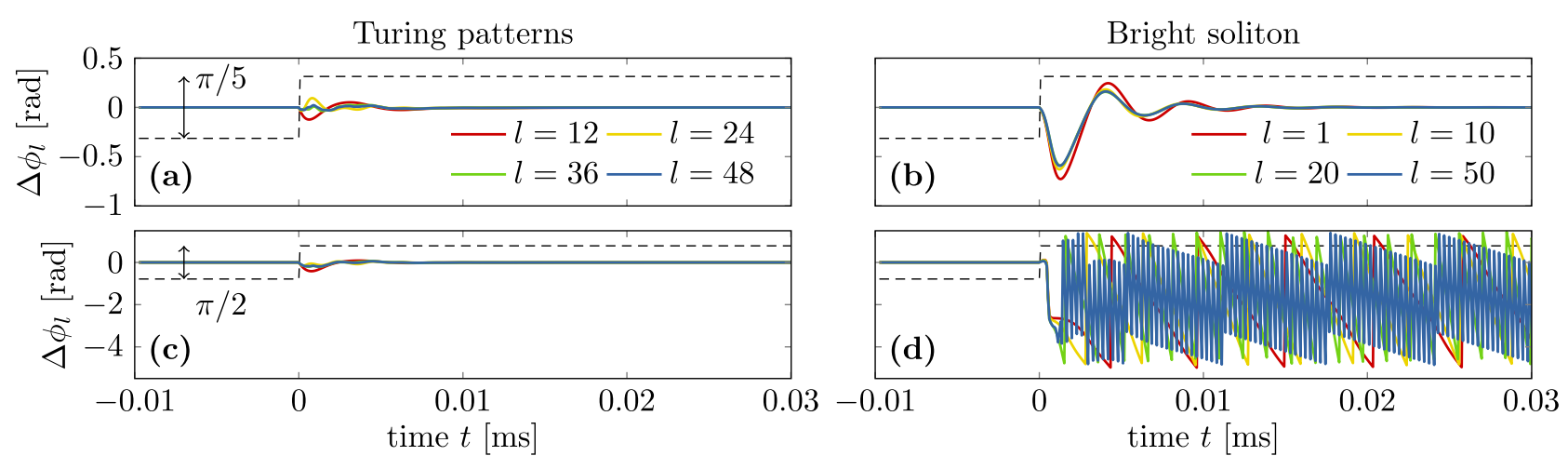

Fig. 6. Variation of the relative phases, $\Delta \phi_{l}$, of different spectral modes of the Kerr combs while the pump signal undergoes an abrupt phase transition. (a) and (c) Case of the Turing pattern of Fig. 3(a). (b) and (d) Case of the bright soliton comb of Fig. 3(b). At $t=0$, the phase of the pump is shifted by $\pi / 5$ in (a) and (b) and by $\pi / 2$ for (c) and (d). In the $\pi / 5$ phase shift case, oscillations in the relative phases of the comb's lines are observed, but the phase locking returns to its previous state and value. The amplitude and duration of these oscillations are much larger in the case of the bright soliton compared to the Turing patterns case. In the $\pi / 2$ phase shift case, while the phase locking of the Turing pattern is maintained, the soliton is destroyed, and thus the phase locking.

for ultrastable microwave generation. However, further investigations are needed in order to generate Turing patterns with frequency spacings below $50 \mathrm{GHz}$, using for example WGMs with larger anomalous dispersion.

Y. C. K. acknowledges financial support from the European Research Council (ERC) through the project NextPhase.

\section{References}

1. T. J. Kippenberg, R. Holzwarth, and S. A. Diddams, Science 332, 555 (2011).

2. P. Del'Haye, S. B. Papp, and S. A. Diddams, Phys. Rev. Lett. 109, 263901 (2012).

3. J. Li, H. Lee, T. Chen, and K. Vahala, Phys. Rev. Lett. 109, 233901 (2012).

4. A. B. Matsko and L. Maleki, Opt. Express 21, 28862 (2013).

5. A. B. Matsko, A. A. Savchenkov, W. Liang, V. S. Ilchenko, D. Seidel, and L. Maleki, Opt. Lett. 36, 2845 (2011).

6. Y. K. Chembo and C. R. Menyuk, Phys. Rev. A 87, 053852 (2013).

7. S. Coen, H. G. Randle, T. Sylvestre, and M. Erkintalo, Opt. Lett. 38, 37 (2013).

8. I. Balakireva, A. Coillet, C. Godey, and Y. K. Chembo, "Stability analysis of the Lugiato-Lefever model for Kerr optical frequency combs. Part II: case of anomalous dispersion," arXiv:1308.2542 (2013).

9. C. Godey, I. Balakireva, A. Coillet, and Y. K. Chembo, "Stability analysis of the Lugiato-Lefever model for Kerr optical frequency combs. Part I: case of normal dispersion," arXiv:1308.2539 (2013).

10. T. J. Kippenberg, S. M. Spillane, and K. J. Vahala, Phys. Rev. Lett. 93, 083904 (2004).

11. A. A. Savchenkov, A. B. Matsko, V. S. Ilchenko, I. Solomatine, D. Seidel, and L. Maleki, Phys. Rev. Lett. 101, 093902 (2008).

12. Y. K. Chembo, D. V. Strekalov, and N. Yu, Phys. Rev. Lett. 104, 103902 (2010)

13. Y. K. Chembo and N. Yu, Phys. Rev. A 82, 033801 (2010).

14. A. Coillet, I. Balakireva, R. Henriet, K. Saleh, L. Larger, J. Dudley, C. Menyuk, and Y. K. Chembo, IEEE Photon. J. 5, 6100409 (2013).

15. A. B. Matsko, A. A. Savchenkov, D. Strekalov, V. S. Ilchenko, and L. Maleki, Phys. Rev. A 71, 033804 (2005).

16. T. Hansson, D. Modotto, and S. Wabnitz, Phys. Rev. A 88, 023819 (2013).

17. M. R. E. Lamont, Y. Okawachi, and A. L. Gaeta, Opt. Lett. 38, 3478 (2013). 